\title{
Implementation of carbon nanomodification for sorption materials
}

\author{
Alexander Babkin ${ }^{1}$,Elena Neskoromnaya ${ }^{1}$, and Irina Burakova ${ }^{1, *}$ \\ ${ }^{1}$ Tambov State Technical University, Department "Technology and Methods of Nanoproducts Manufacturing", \\ 392000, 106 Sovetskaya Str., Tambov, Russia
}

\begin{abstract}
The article addresses the urgent task of improving the adsorption capacity and expanding the scope of application for commonly used industrial sorbents - activated carbons and synthetic zeolites. Among a variety of methods for modifying these sorbents, more attention is now being given to techniques that employ carbon nanomaterials. This is due to the unique properties of nanostructures - developed surface, availability of active functional groups, etc. In the present work, the classic materials - NWC coconut shell activated carbon and synthetic $\mathrm{NaX}$ zeolite - were chosen as initial sorbent samples to be modified. The authors developed a process flowsheet for the carbon nanomodification, which contains the following main stages: preparation of a catalytic mixture solution under given temperature conditions, impregnation of porous materials using the obtained solution, and drying and synthesis of carbon nanotubes via chemical vapor deposition. The proposed technological line consists of a reactor for synthesis of carbon nanotubes, the patented design of which will allow for simultaneously modifying in an effective way different types of materials. As a result, the layer of carbon nanostructures, the quality of which can be varied by changing the conditions of the modification procedure, is formed on the substrate surface.
\end{abstract}

\section{Introduction}

The problems of fine purification of aqueous media, regeneration of wastewater and integrated water treatment for high-tech industries are relevant and due to ever more stringent modern requirements. A promising route for developing effective sorbents is their modification with carbon nanomaterials, in particular, carbon nanotubes (CNTs). The most common sorbents are activated carbon $(\mathrm{AC})$ and zeolites, the modification of which substantially increases their adsorption activity [1-18].

As it is known, the $\mathrm{AC}$ consisting of a lot of randomly arranged graphite microcrystals is usually employed in the processes of purification and separation of liquids and gases (vapors) for adsorption of organic substances.

The zeolites represent natural or synthetic minerals aqueous aliminosilicates containing oxides of alkali and alkaline earth metals.

Despite the variety of sorbents used, many of them do not satisfy the entire set of requirements established for materials of this type. In this regard, the search for and development of novel sorption materials are ongoing.

Industrial sorbents must meet the following requirements: have a great sorption capacity and high mechanical strength, possess high selectivity, ability to regenerate and stability of sorption properties under long-term operationconditions, be non-toxic, noncorrosive, and inexpensive.
At present, a number of new sorption materials are being developed, the production of which has not yet been mastered on an industrial scale. Materials with a modified porous space and various kinds of composites may be attributed to such sorbents. The modification of sorbents promotes the expansion of their application areas, as well as an increase in their adsorption selectivity.

Depending on the application field, there exist different methods for modifying the AC: acid/base treatment, ammonium compound treatment, impregnation using polymer solutions; temperature treatment; plasma treatment; exposure to microwave radiation; ozonation; impregnation using metals; and modification using carbon nanomaterials. As a result of the modification, the surface reactivity, chemical, physical and structural properties of the AC change [1$14]$.

Among the methods for modifying the zeolites, the following ones can be pointed out: composition change during crystallization; ion exchange; introduction of easily adsorbed impurities into the zeolite crystal; decathionization; dealumination; modification using polymers; and modification using carbon nanomaterials [15-18].

As can be seen, for both the AC and zeolites, the modification with carbon nanomaterials is used. The application of CNTs is due to their high adsorption characteristics, the possibility of changing the surface chemistry, and the structural features of the nanometer range which are determined by synthesis method and parameters. Chemical vapor deposition (CVD) with the

\footnotetext{
Corresponding author: iris tamb68@mail.ru
} 
use of metal oxide catalysts appears to be a widespread industrial method of CNT production, which makes it possible to obtain CNT structures over substrates. The most important effect on the nanotube structure is provided with the technology of preparation of metal oxide catalysts and chemical nature thereof. The determination of the dependence of the nanotube properties on the parameters of the catalysts used allows to obtain CNTs with adjustable characteristics (length, diameter, degree of defectiveness, etc.) for surface modification of porous substrates [19-25].

Considering the aforementioned, the aim of the present research is to develop a carbon nanomodification process for industrial sorbents used for purification of aqueous media.

\section{Materials and Methods}

In the modification process, NWC industrial coconut shell $\mathrm{AC}$ and synthetic $\mathrm{NaX}$ zeolite were used as substrates.

Electron images of nanomodified materials were obtained via scanning electron microscopy (SEM) on a
Neon 40 two-beam instrument (Carl Zeiss, Jena, Germany).

\section{Modification Procedure}

The implementation of the experimental-industrial (pilot) production of nanomodified materials based on the $\mathrm{AC}$ and zeolites is possible within the technological line, the flow diagram and elemental composition of which are presented in Fig. 1.

The initial components (nickel, cobalt and magnesium nitrates, and citric acid) enter the reactormixer RM1 from the bunkers B1, B2, B3 and B4 through the dispensers D1, D2, D3 and D4. Ethylene glycol comes into the RM1 from the reservoir R1 by means of the dispensing pump DP.

Distilled water is obtained in the distiller DIS and routed into the reservoir R2. From the R2, the distilled water is supplied into the RM1 using the dispensing pump DP, where all the catalyst components are mixed and treated at $80^{\circ} \mathrm{C}$.

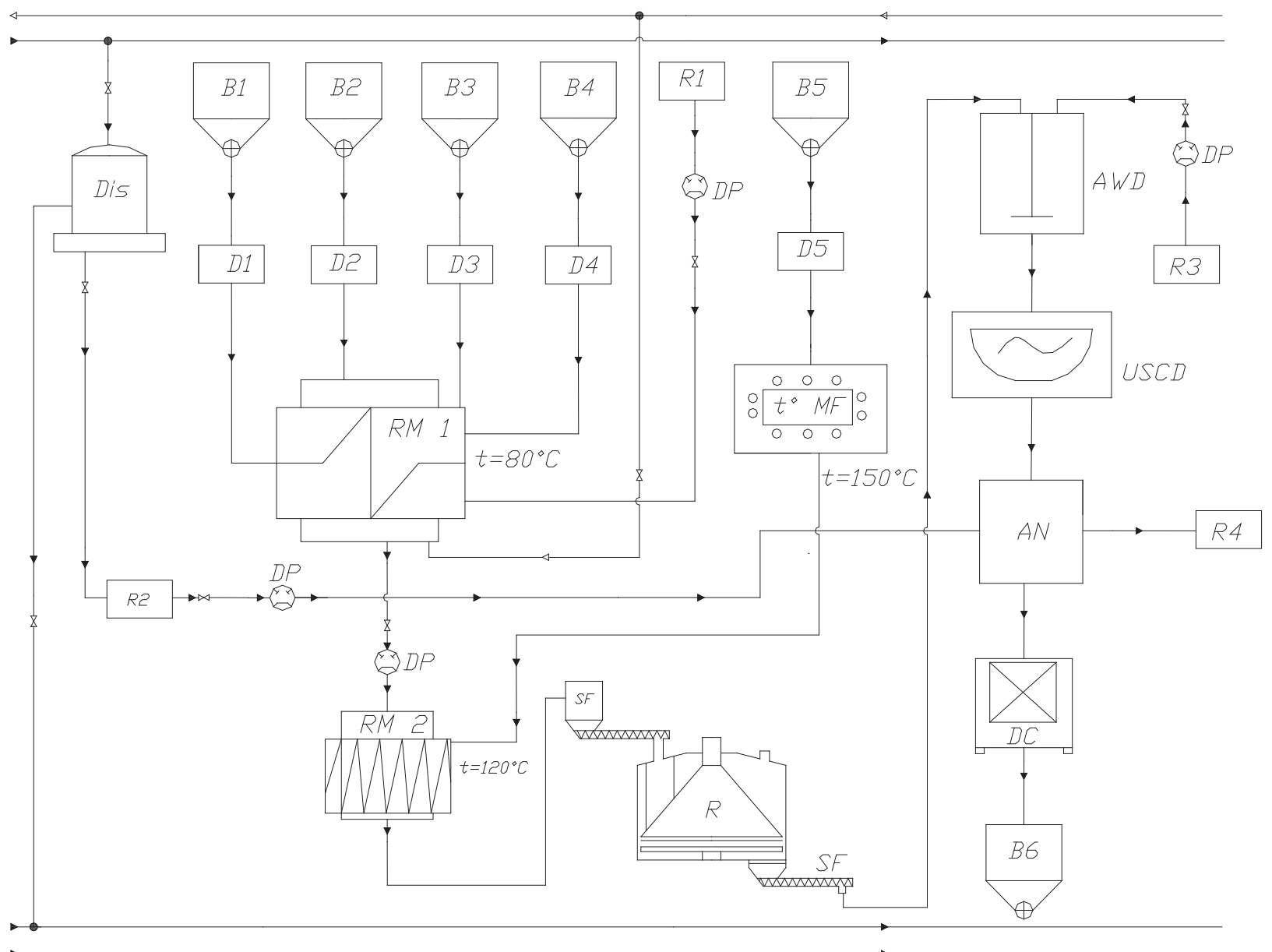

Fig. 1. A flow diagram and elemental composition of the technological process of nanomodified sorbent production. 
The solution is heated in indirect steam. The temperature inside the reactor is raised up to $140{ }^{\circ} \mathrm{C}$. From this reactor, the xerogel formed is routed through the dispensing pump DP into the auger reactor-mixer RM2.

The sorbent comes into the muffle furnace MF (preheated to a temperature of $150{ }^{\circ} \mathrm{C}$ ) from the bunker B5 through the dispenser D5. From the MF, it is supplied into the RM2, where the porous substrate is impregnated with a catalyst solution at $120^{\circ} \mathrm{C}$. Then, the impregnated sample comes into the batch reactor $\mathrm{R}$, where the carbon nanomaterial is synthesized over the substrate surface via catalytic pyrolysis of hydrocarbons.

After that, the nanomodified material is sent to the acid washing device AWD for washing from impurities of amorphous carbon and catalyst particles. Into this device, nitric acid comes from the reservoir R3 through the dispensing pump DP.
Next, the material passes through the ultrasonic cleaning device USCD, and then it enters the drying chamber DC through the acid neutralizer AN. From the $\mathrm{AN}$, the spent acid is withdrawn into the reservoir R4. The obtained nanomodified material is routed into the bunker B6 for storage.

To implement the CNT synthesis process over the impregnated sorbent samples, the design of an upgraded reactor was developed, which allows for increasing the productivity of this stage due to more rational use of the working area of the device.

In the reaction zone of the reactor, there is a device that makes it possible to modify several types of materials: fibrous, granular, and gradient-porous inorganic plates (Fig. 2). The upgraded reactor comprises a housing consisting of the bottom 1 and the lid 2 connected with the sealing unit 3 .

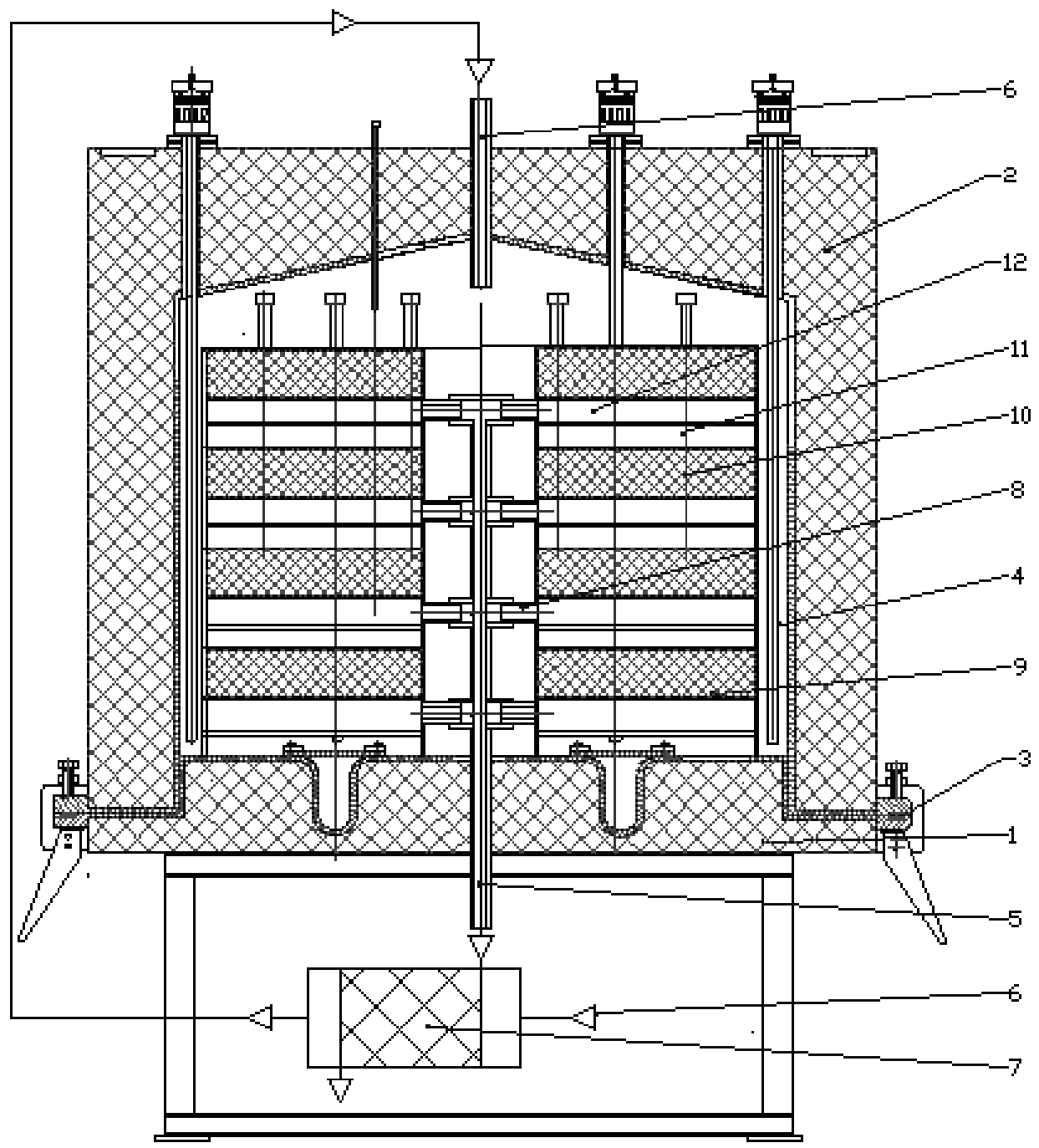

Fig. 2. The design of an upgraded reactor for nanocarbon modification of filter materials ( 1 - bottom, 2 - lid, 3 - sealing unit, 4 - heaters, 5 - gaseous pyrolysis product extraction pipe, 6 - hydrocarbon gas supply pipe, 7 - heat exchanger, 8 - branch pipe (nozzle), 9 deposition chamber, 10 - substrate, 11 - skirt, 12 - reservoir). 
On the lid 2, inside the housing, the heaters 4 and the gaseous pyrolysis product extraction pipe 5 are installed, and on the bottom 1 , hydrocarbon gas supply pipe 6 is mounted.

The hydrocarbon gas supply pipe 6 and the gaseous pyrolysis product extraction pipe 5 are connected together with the heat exchanger 7 installed outside the reactor. On the bottom 1, the deposition chambers 9 are mounted. At the outlet, they are connected with the gaseous pyrolysis product extraction pipe through the branch pipes (nozzles) 8. At the inlet of those chambers, the substrates 10 made of a porous material [26] impregnated with a solution of liquid phase precursors of the CNT synthesis catalyst are placed.

The technical result provided with the reactor lies in obtaining nanomodified materials by depositing on the carbon material impregnated with the catalyst solution.

The proposed device operates as follows: when the lid is up, the deposition chambers and the substrates are installed on the bottom. The chambers are equipped with the substrates in the following way - when using the substrates in the form of granules, the latter are loaded into pallets with a mesh bottom. The substrates in the form of disks are mounted in the lid sockets and fixed with rings. The substrates in the form of sheets are laid on the lattice lid and fixed with frames. The nozzles are connected to the gaseous pyrolysis product extraction pipe. Then, the lid is installed on the bottom, and the reactor is hermetically sealed using the sealing units. Before operation, the reactor cavity is purged with argon through the hydrocarbon gas supply pipe, while the reactor air is removed through the gaseous pyrolysis product extraction pipe.

The pyrolysis of the hydrocarbon gas is carried out at a temperature of $600-700{ }^{\circ} \mathrm{C}$. During this process, the hydrocarbon gas passes through the perforation of the skirts 11 and the substrate 10 into the reservoir 12, and is discharged into the gaseous pyrolysis product extraction pipe 5 through the nozzles 8 . The exhausted hot gases enter the heat exchanger 7, where the heat is supplied into the hydrocarbon gas supply pipe 6.

The results of the implementation of the nanomodified sample synthesis are presented in Figs. 3 and 4 .

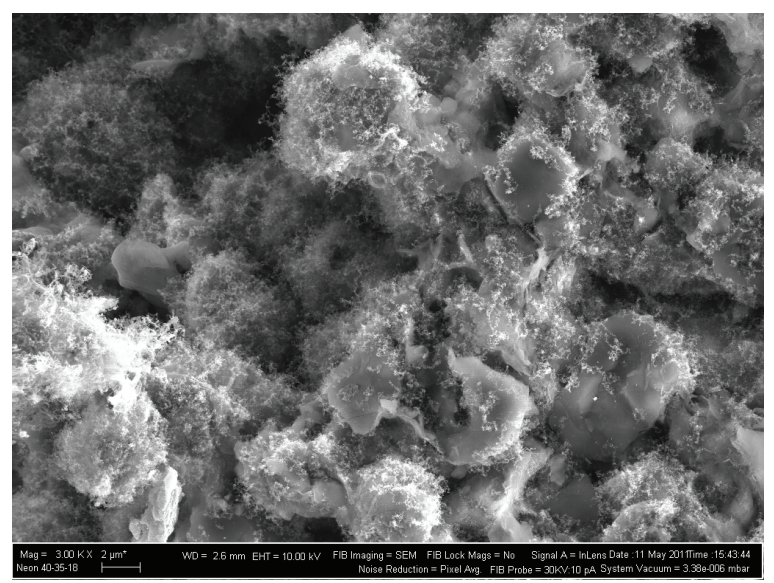

Fig. 3. A modified surface of the NaX zeolite.

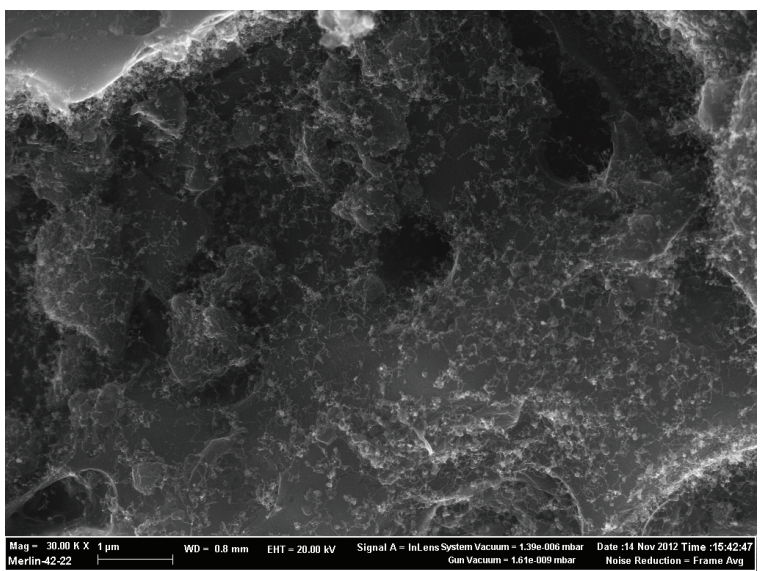

Fig. 4. A modified surface of the NWC coconut shell activated carbon.

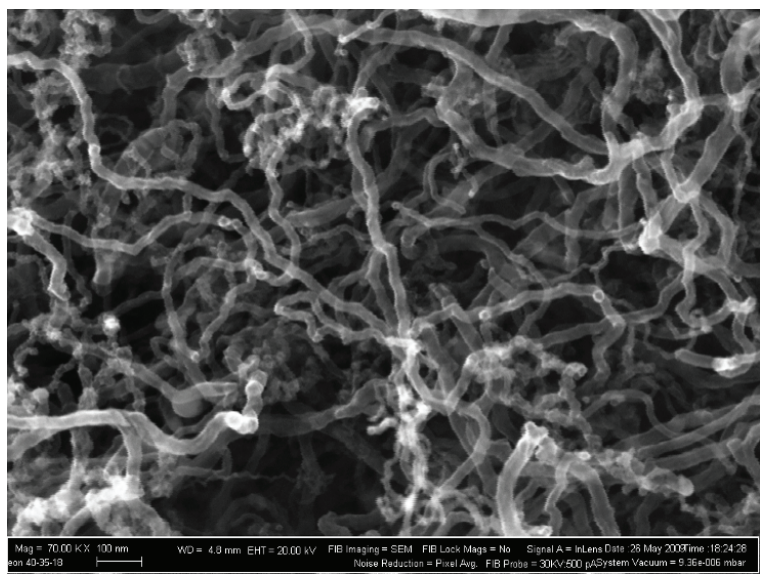

Fig. 5. Morphology of the CNT layer.

Fig. 3 demonstrates the synthetic $\mathrm{NaX}$ zeolite, the surface of which is modified with CNTs. In this figure, individual zeolite granules coated with the CNTs can be observed. The nanotube diameter is $15-30 \mathrm{~nm}$. Besides, the catalyst particles with a size of $15-100 \mathrm{~nm}$ are available in the material.

Fig. 4 shows the structure of the NWC coconut shell $\mathrm{AC}$ with pores of 1-2 microns. It can be seen that the nanomodification resulted in uniform coverage of the CNTs over the AC surface. The nanotube diameter is 15$50 \mathrm{~nm}$, and the catalyst particle size is also $15-50 \mathrm{~nm}$ (Fig. 5).

\section{Conclusion}

Thus, the authors have implemented the formation of a nanotube layer (Patent $106253 \mathrm{RF}$ ) in a porous space of commonly used sorbent materials for developing nanostructured sorbents. The proposed technological solution provides obtaining a uniformly distributed array of nanotubes with adjustable parameters.

The adsorption of organic and inorganic compounds from aqueous solutions appears to be the main trend in using modified materials.

By varying the synthesis mode parameters, as well as by changing the chemical composition of the catalytic 
mixture, it is possible to obtain the CNT layer with a given quality on the surface of porous substrates. This fact can expand the field of application for nanomodified materials.

The research was funded by the Ministry of Education and Science of the Russian Federation (Project No. 16.1384.2017/PCh).

\section{References}

1. Yunhai $\mathrm{Wu}$, Yajun Wen, Jianxin Zhou et.al., ESPR, 20, 4 (2013)

2. Lo Sheng-Fong, Wang Song-Yung, Tsai Ming-Jer, Lin Lang-Dong, Chem. Eng. Res. Des., 90, 9 (2012)

3. Sato Sanae, Yoshihara Kazuya, Moriyama Koji, Machida Motoi, Tatsumoto Hideki, Appl. Surf. Sci., 253, 20 (2007)

4. B. Saha, M.H. Tai, M. Streat, Chem. Eng. Res. Des., 2003. 81, 10 (2003)

5. E. Erdem, N. Karapinar, R. Donat, J. Colloid Interface Sci., 280 (2004)

6. P. Chingombe, B. Saha, R.J Wakeman, Carbon, 43 (2005)

7. Amalina Md. Dali, Ahmmed Saadi Ibrahem, Abdul Hadi, J. Pur. Util. Reac. Environ., 1, 5 (2012)

8. Chun Yang Yin, Mohd Kheireddine Arou, Wan Mohd Ashri Wan Daud, Sep. Purif. Technol., 52, 3 (2007)

9. Xuemei Ren, Changlun Chen, Masaaki Nagatsu, Xiangke Wang, Chem. Eng. J., 170 (2011)

10. S. Vinitnantharat, W. Rattanasirisophon, Y. Ishibashi, Water Sci Technol., 55, 5 (2007)

11. Rachel RibeiroVieira Azzi Rios, Dênio Eduardo Alves, Ilza Dalmázio, Sílvio Fernando Vargas Bento, Claudio Luis Donnici, Rochel Monteiro Lago, Mater. Res., 6, 2 (2003)

12. W. Shen, Z. Li, Y. Lieu, Rec. Pat. Chem. Eng., 1 (2008)

13. M.S. Shafeeyan, W.M. Wan Daud, A. Houshmand, A. Shamiri, J. Anal. Appl. Pyrolysis, 89 (2010)

14. G.G. Stavropoulos, P. Samaras, G.P. Sakellaropoulos, J. Hazard. Mater., 151 (2008)

15. C.R. Oliveira, J. Rubio, Miner. Eng., 20 (2007)

16. G. Ciobanu, D. Ignat, G. Carja, S. Ratoi, C. Luca, Chem. Bull. "POLITEHNICA" Univ. (Timişoara), 53, 67 (2008)

17. Yousheng Tao, Hirofumi Kanoh, Lloyd Abrams, Katsumi Kaneko, Prep. Char. Appl. Chem. Rev., 106 (2006)

18. A. H. Janssen, I. Schmidt, C. J. H. Jacobsen, A. J. Koster, K. P. de Jong, Microporous and Mesoporous Mater., 65, 1 (2003)

19. Ren Xuemei, Chen Changlun, Nagatsu Masaaki, Wang Xiangke, Chem. Eng. J., 170 (2011)
20. Tsu-Wei Chou, Limin Gao, Erik T. Thostenson, Zuoguang Zhang, Joon-Hyung Byun, Compos. Sci. Technol., 70 (2010)

21. Mohamed Abdel Salam, Mohamad S.I. Makki, Magdy Y.A. Abdelaal, J. Alloys Compd., 509, 5 (2011)

22. M. Bahgat, A.A. Farghali, W.M.A. El Rouby, M.H. Khedr, J. Anal. Appl. Pyrolysis, 92, 2 (2011)

23. Savage Nora, Diallo Mamadou S., J. Nanopart. Res., 7 (2005)

24. D.V. Tarov, T.V. Gurova, I.N. Shubin, Vestnik TSTU, 361 (2015)

25. A.G. Tkachev, D.V. Tarov, V.P. Tarov, I.N. Shubin, Vestnik TSTU, 503 (2016)

26. A.E. Burakov, Yu. A. Kobcheva, E.A. Burakova, I.V. Ivanova, A.G. Tkachev Patent of the Russian Federation 106253 U1, № 19 (2011) 